Archives

40 | 2007

Dépendance(s)

\title{
Les religieuses espagnoles au siècle d'or
}

Entre dépendance sociale et autonomie spirituelle

Isabelle Poutrin

\section{(2) OpenEdition}

Journals

Édition électronique

URL : http://journals.openedition.org/ccrh/3339

DOI : $10.4000 /$ ccrh.3339

ISSN : $1760-7906$

Éditeur

Centre de recherches historiques - EHESS

Édition imprimée

Date de publication : 25 avril 2007

Pagination : 51-65

ISSN : 0990-9141

\section{Référence électronique}

Isabelle Poutrin, «Les religieuses espagnoles au siècle d'or », Les Cahiers du Centre de Recherches Historiques [En ligne], 40 | 2007, mis en ligne le 12 octobre 2011, consulté le 19 avril 2019. URL : http:// journals.openedition.org/ccrh/3339; DOI : 10.4000/ccrh.3339

Ce document a été généré automatiquement le 19 avril 2019

Article L.111-1 du Code de la propriété intellectuelle. 


\section{Les religieuses espagnoles au siècle d'or}

Entre dépendance sociale et autonomie spirituelle

Isabelle Poutrin

1 Les autobiographies spirituelles des femmes espagnoles des XVIe et XVII siècles sont, en grande partie, des textes rédigés sur ordre dans le cadre d'une relation de direction spirituelle. À l'origine de ces textes se trouve l'injonction d'écrire donnée par un confesseur à sa pénitente, une religieuse contemplative ou plus rarement une beata (femme appartenant à un Tiers ordre) qui présentait la particularité de connaître des états spirituels hors du commun tels que des extases ou des visions mystiques. Pour les historiens, la relation d'obéissance et de subordination entre les religieuses et leurs confesseurs aurait pu être le point de départ d'une ample réflexion sur la notion de dépendance, abordé sous l'angle tant institutionnel que psychologique ou anthropologique. Or on peut constater que ce concept n'a pas été mobilisé dans ce domaine, les relations entre les religieuses mystiques et leurs directeurs de conscience étant analysées en d'autres termes, dans des champs historiographiques spécifiques. Il nous faut donc en premier lieu présenter ces sources et dresser un bref état de la question, avant de montrer l'articulation entre les deux institutions, la famille et l'Église, qui gouvernaient les religieuses espagnoles, et de nous interroger sur les conditions d'un éventuel désir d'affranchissement personnel chez les religieuses mystiques. L'approche qui est présentée ici est donc sociale et institutionnelle avant tout, car il nous semble qu'elle est le préalable indispensable aux autres types de questionnement concernant la dépendance, que ce soit dans le domaine de l'anthropologie, de la psychologie ou de l'analyse des relations entre confesseurs et religieuses sous l'angle du gender.

\section{Les écrits autobiographiques des mystiques : de I'hagiographie aux approches féministes}

2 Parmi ces écrits autobiographiques féminins, le Livre de la vie de Thérèse d'Avila (vers 1560) reste le plus connu, après avoir été pendant longtemps pratiquement le seul à 
retenir l'attention. Mais à l'époque de la Réforme catholique et même de ce qu'on appelle la " pré-réforme ", commencée en Espagne dès la fin du XVe siècle, nombreuses ont été les femmes à recevoir de leur confesseur l'ordre de coucher sur le papier le récit de leur vie et de leurs expériences intérieures. Il ne s'agissait pas à proprement parler de produire une autobiographie, mais avant tout de confesser les insignes faveurs reçues du Ciel depuis l'enfance, les visions et les extases, les révélations divines, les tentations démoniaques et les divers obstacles spirituels et matériels rencontrés dans l'accomplissement d'un projet de sainteté ${ }^{1}$. L'ordre d'écrire que le confesseur donnait à sa pénitente relevait théoriquement d'une pratique traditionnelle de la direction spirituelle, celle du discernement des esprits - ce domaine de la théologie mystique qui consiste à discerner, entre des phénomènes spirituels sortant de l'ordinaire, ce qui relève de l'intervention divine, ce qui provient d'une action du démon ou de l'imaginaire humain. Nombre d'écrits autobiographiques et de biographies de mystiques, à commencer par le Livre de la vie de Thérèse d'Avila, indiquent que l'ordre d'écrire donné par le confesseur est inspiré par la nécessité d'y voir plus clair, de vérifier l'origine et l'authenticité des phénomènes mystiques extraordinaires. Par l'ordre d'écrire, le confesseur mettait à l'épreuve le lien d'obéissance par lequel la pénitente était assujettie à son directeur spirituel. En effet, la vertu d'humilité commandait aux religieuses de ne pas désirer révéler l'intimité de leur relation avec le Seigneur; mais l'obéissance aux ordres du confesseur devait l'emporter, et cette vertu d'obéissance était considérée comme l'un des signes essentiels d'une vie spirituelle authentique.

3 Ces textes autobiographiques sur ordre ont été pris en compte dans deux champs historiographiques très différents. Le premier, le plus ancien, est celui de l'histoire de la spiritualité et de la mystique comme branche de l'histoire de l'Église. Le caractère hagiographique des études consacrées aux religieuses mystiques dans les ouvrages en langue espagnole est certes moins prononcé aujourd'hui qu'au XVII siècle et, surtout, le souci d'établir rigoureusement les faits historiques y est sensible, qu'il s'agisse ou non d'appuyer une procédure de canonisation. Mais les éventuels conflits d'intérêts entre les personnages - qui pouvaient surgir par exemple, au moment où une religieuse entreprenait sur l'injonction divine de conduire son monastère à l'abandon des pratiques mondaines et à l'adoption d'un mode de vie des plus rigoureux, ou lorsque la communauté religieuse craignait que les extases de la religieuse ne soient un signe de possession démoniaque - sont atténués ou contournés par le récit des événements : dans les biographies ecclésiastiques, tout concourt finalement à la reconnaissance de la sainteté de l'héroïne ${ }^{2}$. Dans cette perspective, les écrits autobiographiques féminins ont été valorisés en fonction de leur contenu doctrinal, comme l'expression d'une connaissance suréminente de Dieu, effet des grâces reçues par les religieuses auteurs de ces textes. L'ordre d'écrire délivré par le confesseur apparaît seulement comme un préalable, nécessaire du point de vue de la situation de la religieuse mystique dans les hiérarchies humaines, alors qu'elle se trouve projetée soudain, par l'union de son âme avec Dieu, dans un ordre hiérarchique surnaturel. Ainsi, l'analyse des liens sociaux en termes de dépendance n'est pas pertinente dans la perspective hagiographique. L'obéissance envers les supérieurs religieux et le directeur spirituel est une voie vers la sainteté, et non un obstacle à l'épanouissement individuel. C'est contre le démon que la religieuse mystique mène son combat, ou contre des groupes hostiles qui s'en font les instruments pour contrarier le dessein de Dieu. Même en butte à son entourage, elle n'est 
pas censée s'élever contre des contraintes sociales ni avoir en vue sa propre émancipation.

4 Pourtant, dans quelle mesure l'ordre d'écrire du confesseur rencontrait-il les aspirations intimes de sa pénitente à prendre la plume ? C'est ici qu'il faut évoquer le second champ disciplinaire à avoir pris en considération les écrits autobiographiques des femmes mystiques, c'est-à-dire l'histoire littéraire sur son versant féministe. L'étude de ces textes a ouvert de nouvelles perspectives à l'histoire des femmes, des relations entre les sexes, de l'élaboration sociale des catégories du féminin et du masculin bref, de ce qu'on appelle l'histoire du genre (gender). Il s'agissait alors de rechercher dans ces écrits une « écriture féminine ",

un langage de femmes spécifique qui pouvait exprimer une identité féminine et devenir un moyen d'accéder à l'autorité et à l'indépendance par rapport à la domination masculine ${ }^{3}$.

5 Il était paradoxal de lire des stratégies d'affirmation de soi, dans des textes produits dans le cadre d'une relation fondée sur l'obéissance à un directeur spirituel, et, de fait, le caractère oppressif de la relation entre confesseur et pénitente n'a pas été ignoré. Mais l'intérêt des chercheurs s'est centré de préférence sur les cas qui, comme celui de Thérèse d'Avila, suggéraient que l'auteur du texte était parvenu à concilier la démonstration de son humilité (nécessaire afin de faire la preuve de son orthodoxie dans un système qui interdisait la participation des femmes à l'enseignement de la théologie) avec l'affirmation de son autorité ${ }^{4}$. La notion d'autorité d'ailleurs, plus que celle d'émancipation, a été placée au centre de ces recherches. Avec le développement aux États-Unis des institutions universitaires consacrées à l'histoire des femmes, il s'agissait de produire de nouvelles héroïnes positives, pionnières, en quelque sorte, du combat des femmes pour leur libération. Ne pas s'appesantir sur les formes de dépendance pesant sur les religieuses mystiques, c'était aussi sortir d'une histoire des femmes à tendance victimaire qui avait été celle des premiers temps de l'histoire féministe, et qui déclinait justement lorsque les études sur l'écriture féminine conventuelle ont pris leur essor.

6 Ce champ de recherches demeure encore largement ouvert, car l'abondance des sources rend possible la multiplication des études de cas et les processus d'élaboration des textes peuvent être étudiés dans toute leur complexité. Les développements récents tendent à mettre en valeur les cas de collaboration entre l'auteur et son confesseur dans la rédaction des écrits autobiographiques, la notion de genre fournissant toujours la grille d'analyse des sources. Ainsi, constate Alison Weber à propos de Thérèse d'Avila, la relation asymétrique entre " une religieuse femme et un clerc homme » (a female religious and a male cleric) pouvait être une relation fructueuse : même dans les conditions de la culture patriarcale catholique de la période moderne, c'est grâce à l'interaction entre des femmes et des hommes que les textes écrits par des femmes accédaient à l'existence puis, parfois, à une très large reconnaissance par la société

7 On le voit, l'étude de ces écrits féminins a privilégié l'approche biographique, tant du côté de l'histoire religieuse que dans la réflexion sur le genre. Le face-à-face de la « religieuse femme et du clerc mâle " s'inscrit dans une micro-histoire où les interactions entre individus sont finement analysées. À ce niveau d'observation, il est possible de voir que certaines femmes parvenaient à prendre de l'ascendant sur leur entourage masculin et féminin en raison de leur réputation de sainteté. Certaines, très rares, accédèrent à la publication sous leur nom, il est vrai à titre posthume, et ce malgré l'interdiction qui 
pesait sur la parole publique des femmes dans l'Église. Pour autant, est-il juste de considérer ces histoires comme des cas de réussite dans l'affirmation de soi et dans l'acquisition d'une position d'autorité dans un monde dominé par les hommes ? S'agit-il pour autant d'une expérience émancipatrice (et par rapport à qui)?

\section{Le poids de l'institution : familles et couvents}

8 Il est nécessaire de replacer le petit nombre des religieuses mystiques dans les mouvements d'ensemble qui affectèrent les religieuses contemplatives à l'époque de la Réforme catholique. Même si cela semble une évidence, il faut souligner le poids de l'institution, qu'elle soit familiale ou ecclésiastique, dans la vie de ces femmes. À l'époque moderne, hommes et femmes étaient liés par des «liens personnels de tous ordres, principalement des liens familiaux, de parenté, de lignage, de clan, d'amitié, de clientèle » ${ }^{6}$. Les filles, qui se trouvaient toujours dans une position inférieure dans la hiérarchie familiale, étaient vouées à passer d'une dépendance à l'autre : des parents, au mari ou au couvent. C'est ce passage qu'il nous faut approfondir à présent.

9 Car la relation qui unit familles et couvents est complexe. Les volontés des parents décidaient de façon primordiale de l'état donné aux enfants ; dans les familles aisées, en Espagne comme en Italie ou en France aux XVI et XVIIesiècles, les filles en surnombre étaient bien souvent placées au couvent, car le célibat féminin était inconcevable quand il n'était pas encadré par le statut de religieuse. Cependant, les liens du sang formaient des devoirs et des obligations qui ne s'éteignaient pas avec l'entrée au couvent. Les origines familiales et sociales des religieuses demeuraient l'un des éléments importants de leur identité, même après de longues années dans le cloître, ce dont témoignent bien des autobiographies : les confesseurs commanditaires des récits de vie ne négligeaient pas de demander à leurs pénitentes d'identifier leurs parents ${ }^{7}$. Les indications données permettent de cerner le milieu social de la plupart des auteurs de ces textes: ce sont en majorité des filles de letrados (ces diplômés des universités de droit qui étaient les homologues espagnols des nobles de robe français)et des filles de l'aristocratie,ce qui correspond au recrutement des couvents. Les sentiments d'obligation morale à l'égard des parents et de gratitude pour l'éducation chrétienne reçue, et même la mention de la " pureté de sang » du lignage familial (le fait de ne pas avoir d'ancêtres juifs, musulmans ou hérétiques) : tous ces éléments considérés comme autant de facteurs favorables au projet de sainteté que les religieuses mystiques avaient placé au cœur de leur vie, sont évoqués dans les récits de vie à l'intention des confesseurs. Par exemple la religieuse franciscaine Estefanía de la Encarnación (1597-1665), qui compose le récit de sa vie après seize ans de vie monastique, précise :

Ceux qui m'ont donné le jour étaient très chrétiens et
craignant Dieu, et quant à la qualification de leur
sang, purs. Mon père était de la noblesse de
Bourgogne [...]. Il s'appelait Estevan Guari. Ma mère
naquit à San Martin de Valdeiglesias, bien que la
maison de ses parents fût des Montagnes de Burgos,
des Velázquez y Canales. Elle s'appelait doña María de
la Canal ${ }^{8}$.

Dans l'organisation des couvents féminins telle qu'elle se présente aux XVe et XVI siècles, les liens familiaux étaient primordiaux puisque la commu-nauté des biens avait souvent été abandonnée en raison de difficultés économiques récurrentes. Les bâtiments 
conventuels étaient découpés en "cellules », sorte d'appartements collectifs répartis par groupes lignagers, les religieuses cohabitant sur plusieurs générations (tantes, nièces, sœurs, cousines). Il était d'ailleurs possible de placer une fille au couvent dès la prime enfance afin de mieux la conditionner à la forme de vie qui devait devenir la sienne. Toute une série d'adoucissements dans l'observance de la règle autorisaient le maintien du rang social à l'intérieur du couvent : l'emploi du titre de doña et du nom de famille, la décoration plus ou moins luxueuse des cellules, les bijoux et les parures, les servantes, les distractions aristocratiques telles que le jeu, le théâtre et la musique témoignaient de l'appartenance d'une religieuse à son groupe social d'origine, qui se superposait à l'état d'épouse du Christ.

11 C'est dans ces cellules collectives que s'organisaient les factions qui visaient à assurer l'accès de l'une des femmes de la parenté aux charges de gouvernement (abbatiat, priorat), charges rémunératrices pour la famille de la religieuse concernée: ainsi les élections abbatiales mobilisaient les groupes familiaux à l'intérieur comme à l'extérieur du couvent, les rivalités de pouvoir dans les cloitres prolongeant les stratégies menées au sein des conseils municipaux par les membres du patriciat urbain9. Par ailleurs, la paupérisation des couvents dans la première moitié $d u$ XVI siècle interdisait aux religieuses de rompre avec leur famille, qui conservait son rôle nourricier. C'est ainsi que la dépendance à l'égard de la famille était confirmée et redoublée au sein du couvent, notamment lorsque la tante qui tenait lieu de chef du clan accédait au pouvoir abbatial.

\section{Les couvents féminins à l'heure de la remise en ordre}

Dans le contexte de la restauration de la discipline de l'Église au cours du XVI siècle, et plus encore dans les décennies qui suivirent le concile de Trente achevé en 1563, les couvents féminins furent l'objet de la sollicitude des autorités tant politiques que religieuses, en Espagne plus encore sans doute que dans les pays catholiques voisins. Pour l'Église, il était nécessaire de revaloriser le célibat consacré, dont la valeur spirituelle était niée par les protestants au point qu'ils avaient fermé les monastères. Il fallait donc mettre fin aux scandales qui affectaient certains couvents ouverts aux tentations du monde, et remettre en ordre ces établissements. L'expression de " réforme conventuelle » désigne le processus de restauration de la discipline dans les cloîtres, une entreprise qui allait de pair avec la Réforme catholique qui devait toucher l'Église dans son ensemble. On doit souligner que cette réforme disciplinaire qui affecta le destin de milliers de religieuses ne procédait pas, de la part de l'Église, d'une volonté de persécution misogyne mais d'une logique théologique: il fallait remettre à l'honneur les bonnes œuvres qui étaient, avec la foi en Dieu, la condition du salut. Comme l'assistance aux pauvres ou les prières adressées aux saints, la chasteté des épouses du Christ était une bonne œuvre, un chemin vers Dieu. Il était urgent de s'assurer que les religieuses restaient dans la bonne voie en leur barrant tout accès au monde séculier. Cette préoccupation rencontrait celle des familles attachées à la conservation de leur honneur : mieux contrôler la conduite des religieuses convenait aux familles et aux autorités locales autant qu'à l'Église. De leur côté, les monarques espagnols soutinrent cette entreprise qui leur semblait méritoire aux yeux de Dieu, et favorable à l'ordre public.

13 On peut s'interroger sur l'évolution du lien de dépendance entre les religieuses et leur famille, dans la nouvelle configuration dessinée par la réforme conventuelle. L'élément primordial de celle-ci consistait en l'imposition de la clôture, l'enfermement des 
religieuses contemplatives, en vertu du décret De Regularibus et monialibus adopté lors de la $25^{\mathrm{e}}$ session du concile de Trente et renforcé par des dispositions ultérieures. Pour conduire ce programme, l'autorité sur les couvents féminins fut confiée aux évêques, de préférence aux branches masculines des ordres. Ensuite l'Église s'attacha à distendre les liens des religieuses avec leur famille, par le rétablissement de la communauté des biens dans les couvents, l'obligation d'apporter une dot, le contrôle des élections abbatiales et des communications entre les religieuses et l'extérieur, mesures qui instauraient un régime unique pour les couvents de femmes. Par exemple, la remise en ordre des élections abbatiales était destinée à lutter contre les rivalités des familles de notables au sein des couvents ou contre la monopolisation des charges de commandement par un groupe dominant. La réforme conventuelle pouvait ainsi léser les intérêts particuliers des groupes sociaux dominants. Néanmoins, ces dispositions ne purent pas être appliquées partout avec la même rigueur, parce que les couvents de femmes, pour conserver leur recrutement, devaient conserver également leur fonction sociale de refuge des filles surnuméraires des familles aisées.

14 Par une série de scissions d'ordres religieux et de nouvelles fondations de couvents, on aboutit en Espagne à une situation duale : d'un côté, une élite de couvents réformés et de l'autre, des couvents où les normes nouvelles étaient moins appliquées et où entraient des femmes de santé fragile ou de vocation tiède, voire inexistante. Les établissements réformés sont les mieux connus, car ils ont bénéficié d'une intense promotion : les ordres religieux issus de ce processus (souvent appelés "déchaussés", la sandale étant le symbole de l'austérité retrouvée) accomplirent en effet un travail historiographique intense pour se montrer comme d'efficaces laboratoires de sainteté. La réforme conventuelle fut soutenue par les rois d'Espagne (l'austère Philippe II, le dévot Philippe III et même l'inquiet Philippe IV) et par les grandes familles aristocratiques. Les bailleurs de fonds qui finançaient la fondation et l'entretien d'un couvent y gagnaient des places garanties pour les filles de leur famille, et les prières perpétuelles de la communauté.

Jusqu'à l'entrée au couvent, le sort des jeunes filles restait lié à la capacité de leur famille à obtenir une place, si possible dans les meilleurs établissements. Pour entrer dans les nouveaux couvents les plus prestigieux, par exemple celui des Augustines récollettes de l'Incarnation, fondé en 1611 sous le patronage de Philippe III, la concurrence était rude, il fallait des soutiens à la cour ${ }^{10}$. Entrées en religion, il arrivait encore souvent aux religieuses de retrouver dans la communauté leur sœur ou d'autres filles de leur famille, mais les liens familiaux à l'intérieur et à l'extérieur se trouvaient distendus par l'observance de la règle: le silence hors des offices canoniques, la rareté des communications avec un monde extérieur masqué par des grilles, des volets et des voiles noirs, l'imposition d'un nom religieux exprimant les dévotions du couvent ou de l'ordre, l'uniformité de l'habit, le caractère communautaire des espaces tels que le dortoir et le réfectoire ${ }^{11}$. Si ces dispositions avaient pour objet affiché de protéger la chasteté des religieuses, elles visaient avant tout à empêcher toute intrusion du monde aristocratique à l'intérieur de la clôture. La répartition des pouvoirs dans la communauté fut également repensée : à côté de la supérieure chargée de diriger la communauté dans sa vie matérielle, le confesseur, qui était censé assurer la direction spirituelle des religieuses, s'ingéra de plus en plus dans le gouvernement de la communauté au point d'en arriver à doubler la supérieure ${ }^{12}$. 


\section{Les résistances : une réaction aristocratique}

16 Ces changements furent généralement mal accueillis, notamment dans les couvents les plus aristocratiques où les religieuses se mobilisèrent, avec leur famille, pour conserver le mode de vie antérieur à la réforme. Les conflits qui s'ensuivirent sont encore mal connus. Ils apparaissent dans les autobiographies de mystiques et les biographies de saintes religieuses, mais on doit souligner que ces textes ont été produits dans les milieux qui soutenaient la réforme conventuelle, afin, justement, de témoigner de la réussite de cette entreprise et de la capacité de l'Église à produire des saintes dans un monde conventuel revenu à sa vocation première. Dans ces écrits, les religieuses tenantes de l'ancien mode de vie sont présentées comme des femmes égarées et violentes, et le conflit est exprimé dans des termes moraux et non sociaux. L'histoire de doña María Vela y Cueto (1561-1617) en offre un exemple: cette jeune fille, issue de la meilleure noblesse d'Avila, semblait destinée à suivre l'exemple de sa tante, abbesse du monastère des cisterciennes de la ville, et à lui succéder à la tête de cet établissement prestigieux où elle était entrée dès l'âge de quinze ans. Cependant la réforme conventuelle avait déjà touché la ville, où Thérèse d'Avila avait fondé en 1562 un couvent de carmélites déchaussées non sans avoir surmonté de fortes oppositions. Doña Maria se sentit elle aussi appelée à suivre la voie de la prière et de l'ascèse, prenant pour modèle sainte Catherine de Sienne. Dans les années 1596-1610, les moniales de la communauté se dressèrent contre elle, non seulement parce que doña María présentait des états inhabituels, tels que des paralysies, des évanouissements, de longues périodes d'anorexie et des extases, qui pouvaient être des signes d'une emprise démoniaque, mais aussi parce que la religieuse fervente voulait amener les sœurs à adopter l'habit austère, la stricte clôture et l'emploi du temps rigoureux propre aux établissements réformés. Il semble que la communauté se fractionna entre partisans et adversaires de doña Maria, qui fut même examinée par l'Inquisition en 1604 sur la dénonciation de quelques opposants. Le soutien de son confesseur Miguel Gonzalez Vaquero, qui lui ordonna d'écrire le récit de sa vie puis, après sa mort, publia sa biographie, fut essentiel dans la reconnaissance de la sainteté de doña María $^{13}$.

17 Nous pouvons discerner la composante sociale des oppositions qui s'élevèrent contre celle-ci : revenir à l'observance de la règle, c'était pour les moniales renoncer à tous les éléments de confort et de rang qui rendaient supportable une vie monastique qu'elles n'avaient pas toujours choisie. De plus, les exercices ascétiques et les pénitences appartenaient à une spiritualité en vogue parmi les beatas, des femmes souvent de milieux modestes; les carmélites déchaussées venaient de groupes plus élevés dans la société, surtout de la noblesse petite et moyenne. En refusant le passage de leur monastère à la réforme, il semble que les cisterciennes de Santa Ana, issues des familles les plus puissantes et les plus nobles de la ville, aient voulu conserver la distance sociale qui les distinguait des autres dévotes et religieuses. Aussi peut-on interpréter les oppositions à la réforme conventuelle dans les grands monastères, non pas comme la volonté des religieuses de sauvegarder une autonomie individuelle que, de toutes façons, elles n'avaient pas, mais bien plutôt comme une forme de résistance nobiliaire dirigée contre la suppression des groupes de parenté dans les couvents et contre la spoliation des symboles de l'honneur tels que le titre, le nom, le vêtement, les loisirs, les domestiques et le prestige donné par les fonc-tions de direction. Les cas de fuite ou de suicides de 
religieuses relevés en Italie et en Espagne lors de l'imposition de la réforme dans certains couvents, montrent sans doute le désespoir des intéressées, mais on constate aussi, lors de la réforme des couvents de Barcelone entreprise à la fin du Xve siècle, la complicité des familles dans les cas d'évasion ${ }^{14}$.

Toutefois, la réforme conventuelle n'entraîna pas une rupture totale des religieuses avec leur milieu familial. Cette piste n'est pas vraiment explorée par l'historiographie conventuelle, mais on y relève quelques indices. Durant la période d'installation de la réforme, entreprise qui forme la matière des biographies des grandes religieuses fondatrices ou des abbesses réformatrices, le rôle des liens de parenté ainsi que, d'ailleurs, des liens d'amitié personnelle, fut déterminant dans la réussite des supérieures à imposer une discipline plus rigoureuse à leurs consœurs, elles-mêmes issues de couches sociales jalouses de leurs prérogatives : l'exemple des religieuses cisterciennes françaises au XVII siècle montre que l'origine familiale de la supérieure, sa «surface sociale » en quelque sorte, sa capacité à mobiliser ses relations à la cour conditionnait l'étendue réelle de ses pouvoirs dans sa communauté15. En Espagne, il fallut pour rétablir l'ordre au monastère de las Huelgas de Burgos, l'un des plus sélects de Castille, que Philippe III y nomme sa cousine abbesse perpétuelle ${ }^{16}$.

Par la suite, l'entrée au couvent demeura un acte marqué par l'emprise familiale. Certes, selon les prescriptions du concile de Trente, le consentement de la postulante devait être examiné par l'évêque ou son vicaire général en présence d'un notaire. Mais la pratique subsista, dans la noblesse, de placer simultanément plusieurs filles au couvent dès l'enfance. Au XVIII siècle, période marquée en Espagne par le déclin des vocations contemplatives et par les difficultés économiques qui touchaient la plupart des couvents, les pressions familiales pour pousser une fille à entrer au couvent demeurent une réalité attestée par les archives notariales, comme le montre l'étude conduite à Zamora par Francisco J. Lozenzo Pilar: des religieuses ont fait enregistrer devant notaire des témoignages de protestation, confessant qu'elles faisaient profession par crainte et respect filial, des demandes d'annulations de vœux alléguant la contrainte et la violence. Ces demandes étaient toutefois repoussées dans la plupart des cas parce qu'elles étaient présentées après le délai légal de cinq ans après la profession, par ignorance de la loi ou parce qu'il fallait attendre la mort du père ou du tuteur. Des procès témoignent aussi du trucage des professions: ainsi l'affaire de cette novice qui avait prononcé ses vœux à l'intérieur de la clôture (et non à l'entrée), dans l'obscurité et derrière un épais grillage. Attaquée par le représentant de l'évêque, la supérieure du couvent gagna le procès, ce qui montre à quel point la règle du consentement des religieuses pouvait être bafouée ${ }^{17}$. On peut voir dans ces témoignages et ces procédures une tentative des femmes pour recouvrer leur liberté, mais aussi la résistance des familles et des religieux, avec le soutien des autorités, à relâcher leur emprise.

\section{La religieuse mystique : un modèle de sainteté féminine au siècle de la réforme catholique}

Nous avons vu que les liens de dépendance matérielle et affective des religieuses à l'égard de leur famille évoluèrent quelque peu avec la réforme conventuelle, mais qu'ils ne disparurent pas totalement, d'autant que les prescriptions du concile de Trente ne furent pas appliquées dans tous les établissements avec la même rigueur. Certaines dispositions, 
comme celles qui devaient éviter les vocations forcées, semblaient avantageuses pour les religieuses mais leur application heurtait les intérêts convergents des familles et des communautés. D'autres, comme l'imposition de la clôture, rencontrèrent des résistances dont nous avons tenté de dégager les facteurs sociologiques. Il nous faut nous demander à présent si la réforme conventuelle a pu représenter pour les religieuses une forme d'affranchissement à l'égard de leur milieu familial, et un accès à une autonomie individuelle.

21 Posée en ces termes, la question appelle évidemment une réponse négative : si le mode de vie en vigueur dans les couvents réformés diminuait la force de la dépendance par rapport à la famille et au milieu social d'origine, c'était pour mieux asseoir l'autorité des supérieurs religieux. Dans les couvents réformés ou déchaussés, le nombre des religieuses était volontairement réduit (pas plus d'une trentaine) afin de mieux souder la communauté. La prieure était élue pour une période de trois ans par les religieuses: c'était, semble-t-il, la seule occasion pour les femmes de cette époque d'exercer un droit de vote, mais il n'y avait ni acte de candidature, ni « campagne électorale ». Néanmoins la prieure n'avait pas de pouvoir de juridiction sur ses consœurs ; celui-ci était exercé par le supérieur du couvent, qui était soit le supérieur provincial de l'ordre religieux (dans sa branche masculine), soit l'évêque du lieu : tout dépendait du régime juridique qui prévalait lors de la fondation du couvent, le plaçant sous la juridiction de l'évêque ou du supérieur provincial.

Concernant les effets de cette réforme, les appréciations sont très divergentes en fonction du point de vue adopté. Des études récentes menées notamment en France et en Allemagne ont montré par exemple que l'imposition de la clôture dans des établissements où elle n'était pas en vigueur jusque-là, a eu des conséquences néfastes sur l'insertion des communautés dans le tissu social local, et qu'il a fallu bien souvent réorganiser les activités des religieuses pour trouver des moyens de subsistance compatibles avec leur réclusion ${ }^{18}$. Mais dans la littérature religieuse qui fleurit aux XVI et XVII siècles, la clôture est présentée comme la condition expresse de la pratique des vertus propres à l'état religieux et de la recherche de Dieu dans la prière. En enfermant les corps, elle libérait les âmes, les rendait disponibles pour la recherche de la perfection. Ce discours a été reçu par nombre de femmes au temps de la Réforme catholique, comme le montre le succès des couvents de carmélites déchaussées fondées par Thérèse d'Avila, suivies par d'autres instituts créés en Espagne tels que les augustines récollettes (1603), les trinitaires déchaussées (1612) et les mercédaires déchaus-sées (1617).

Les femmes qui entraient dans ces communautés faisaient le choix d'une dépendance totale à l'égard de leurs supérieurs dont le pouvoir, il est vrai, était limité par la règle. Cette dépendance choisie dans un mode de vie très austère, s'opposait à la dépendance subie par les religieuses de vocation plus tiède, dans les couvents où la réforme était médiocrement appliquée. Les religieuses qui faisaient profession dans les couvents de déchaussées s'identifiaient avec les objectifs de la réforme catholique, et elles étaient prêtes à suivre le chemin de la perfection sur les traces de mères fondatrices charismatiques. Tout était prévu pour remodeler la personnalité des religieuses et pour en faire des créatures tournées vers Dieu. Entre les murs du cloître, dans la stricte organisation du temps rythmé par les offices, les religieuses devaient se dépouiller de leur identité sociale et, si possible, des aspérités de leur caractère. L'humilité, plus que la chasteté, était la vertu qu'il fallait acquérir au prix d'une vie de mortifications. Comme la perfection chrétienne exigeait l'anéantissement de toute volonté propre, les religieuses 
animées par une vocation forte s'appliquaient à correspondre à l'idéal qui leur était présenté. Afin de mériter la couronne de gloire dans le Ciel, il fallait sur terre «souffrir pour le Christ", impératif répété de façon obsédante dans les biographies et les autobiographies de religieuses.

Les couvents d'Espagne devaient produire des saintes, pour montrer aux hérétiques de l'Europe entière, à ces Hollandais et Anglais qui défiaient la puissance du Roi Catholique, que les grâces de Dieu se répandaient sans relâche sur la terre d'élection du catholicisme. Les femmes pouvaient prendre leur part dans ce combat, entre les murs du couvent, en pratiquant les vertus jusqu'à l'héroïsme et, surtout, en livrant le récit des faveurs reçues du Ciel. Ainsi, l'abondance des écrits autobiographiques de religieuses en Espagne, à la fin du XVI et au long du XVII siècle, correspond à la mise en œuvre du programme disciplinaire et théologique de l'Église tridentine : programme disciplinaire, comme nous l'avons vu, avec la fondation de couvents réformés et déchaussés. C'est dans ces maisons que fleurissent les écrits autobiographiques rédigés sur ordre ou dans les monastères en voie de réformation ; programme théologique car les débats sur la grâce et le libre arbitre, point de désaccord entre catholiques et protestants, conduisirent non seulement, comme nous l'avons évoqué, à revaloriser la chasteté en tant que bonne œuvre contribuant au salut, mais aussi à réviser la théologie mystique et tout ce qui concernait les charismes donnés par Dieu à un petit nombre d'âmes.

Certains théologiens, espagnols notamment, en vinrent à considérer que l'illumination de l'entendement par la grâce était une opération miraculeuse, qui n'était plus dépendante de la progression dans la vie spirituelle ${ }^{19}$. Sur ces fondements théologiques, une enfant de six mois pouvait entrer en extase, une simple bergère pouvait avoir tous les jours des visions de la Vierge Marie, et des expériences aussi relevées que le mariage mystique ou la stigmatisation pouvaient arriver à d'obscures religieuses pour peu que Dieu les ait choisies et désignées pour être des saintes. L'identification d'un grand nombre de femmes à ces modèles de sainteté, véhiculés par toute une littérature hagiographique, se traduisit par plusieurs générations de religieuses mystiques, émules de Thérèse d'Avila et de Catherine de Sienne ${ }^{20}$.

Dans ce contexte, la commande de récits de vie et d'expériences mystiques, leur publication et leur diffusion s'inscrivaient dans une procédure qui laissait peu de place à l'improvisation. La vie spirituelle des femmes à qui leurs confesseurs commandaient de tels textes, avait fait l'objet de vérifications préalables. Le lien entre le confesseur et sa pénitente reposait sur des bases variables: soit le confesseur était celui de la communauté, et dans ce cas son autorité était garantie par le supérieur du couvent ; soit la religieuse avait pu le choisir, avec l'autorisation de sa supérieure, et dans ce cas le lien reposait sur l'obéissance volontaire de la pénitente à son directeur. Les confesseurs capables de diriger les religieuses les plus remarquables formaient une élite de professeurs de théologie et de dirigeants d'ordres religieux, parfois auteurs d'ouvrages de spiritualité. Pour ces hommes, il était gratifiant de diriger l'âme d'une future sainte et de passer ainsi à la postérité, comme Raymond de Capoue, le confesseur de sainte Catherine de Sienne ${ }^{21}$. Évidemment, cette entreprise comportait des risques, car l'Inquisition, gardienne de l'orthodoxie, réprimait les prétentions à la sainteté quand elles semblaient abusives. Si l'affaire tournait mal, la religieuse et son confesseur étaient condamnés au silence. Le modèle de sainteté mystique commença à être remis en question dans les années 1630 et fut encore plus malmené dans les années 1680, mais il conserva des adeptes jusqu'au milieu du XvIII' siècle ${ }^{22}$. 


\section{Conclusion}

En exposant les conditions sociales et culturelles dans lesquelles ont été produits les récits de vie des mystiques espagnoles, je me suis efforcée de mettre en évidence différentes formes de dépendance : en premier lieu, une dépendance d'ordre juridique et économique qui liait ces femmes à leur famille, puis à l'institut religieux au sein duquel elles prononçaient leurs vœux. Ces deux institutions, la famille et le couvent, entretenaient l'une avec l'autre des relations de collaboration, à l'exception des épisodes de conflits liés à la réforme conventuelle. En prenant le terme de dépendance dans un sens plus large, j'ai voulu montrer à quel point la forme de vie des religieuses déchaussées, de même que le contenu de leurs expériences spirituelles ainsi que la production et la promotion de leurs récits de vie, procédaient des objectifs qui étaient ceux de l'Église catholique entre le milieu du XVI et la fin du XVIe siècle. Dans ce cadre, l'idéal de réussite des religieuses mystiques n'était pas l'autonomie personnelle ou l'acquisition d'une autorité, mais la sainteté. Que ce projet de sainteté ait pu verser, parfois, dans l'imitation formelle des faits et gestes des grandes saintes et dans une forme de rivalité avec elles, est très probable. Toutefois, la référence à l'action divine et les jugements dépréciatifs portés sur soi, omniprésents dans les autobiographies, ne sont pas à mon sens des stratégies consciemment destinées à rendre acceptable la parole féminine aux yeux de lecteurs hommes, mais l'expression d'habitus plus ou moins intériorisés. De nos jours, il peut être difficile d'imaginer que des femmes trouvent leur épanouissement dans l'aliénation de soi et dans l'acceptation d'un conditionnement culturel qui vise à les rendre obéissantes et silencieuses. Sans oublier le contexte spécifique de la Réforme catholique, l'exemple actuel des femmes engagées dans des courants religieux intégristes peut nous fournir un utile point de comparaison pour mieux comprendre les religieuses espagnoles de l'époque tridentine.

\section{NOTES}

1. Isabelle Poutrin, Le voile et la plume: autobiographie et sainteté féminine dans l'Espagne moderne, Madrid, Casa de Velazquez, 1995.

2. Voir par exemple Patrocinio Barriuso Voir par exemple Patrocinio Barriuso, La monja de Carrión, Sor Luisa de la Ascensión Colmenares Cabezón (Aportación documental para una biografía), Madrid, Monte Casino, 1986 ; Eugenio Ayape Moriones, Historia de dos monjas místicas del siglo XVII. Sor Isabel de Jesús (1586 - 1684), Sor Isabel de la Madre de Dios (1614 - 1687), Madrid, Ediciones Augustinus, 1989.

3. Marta V. Vicente et Luis R., Corteguera, « Women in texts : from Language to Representation » in Marta V. Vicente et Luis R. Corteguera (dirs.), Women, Texts and Authority in the Early Modern Spanish World, Aldershot,Ashgate, 2003, p. 1; Leigh Gilmore, Autobiographics : A Feminist Theory of Women's Self-Representation, Ithaca, Cornell University Press,1994. 
4. Alison Weber, Teresa of Avila and the Rhetoric of Feminity, Princeton, Princeton UniversityPress, 1990 ; Sherry M. Velasco, Demons, Nausea and Reistance in the Autobiography of Isabel de Jesus, 1611 1682, Alburquerque, University of NewMexicoPress,1996.

5. Alison Weber, «The Three Lives of the Vida: the Uses of Convent Autobiography " in Marta V. Vicente et Luis R. Corteguera, (dirs.), op. cit., p. 120.

6. José Maria Imizcoz Beunza, «Communauté, réseau social, élites. L'armature sociale de l'Ancien Régime ", in Juan Luis Castellano et Jean-Pierre Dedieu (dirs.) : Réseaux, familles et pouvoirs dans le monde ibérique à la fin de l'Ancien Régime, Paris, CNRS Éditions, Maison des Pays Ibériques, 1998, p. 31-66, en particulier p. 39.

7. Isabelle Poutrin, «L'identité sociale dans les écrits autobiographiques féminins de l'Espagne moderne » in Jean-PierreBardet, et François-JosephRuggiu (dirs.), Au plus près du secret des cœurs? Nouvelles lectures historiques des écrits du for privé en Europe du XVIe au XVIII siècle, Paris, Presse universitaire Paris-Sorbonne, 2005, p. 93-110.

8. « Mandame Vuestra Paternidad diga el estado de mis padres [...]. Los que me dieron naturaleza fueron christianissimos y temerosos de Dios, y, en cuanto a calificación de sangre, limpios. Mi padre fue de lo noble de Borgoña [...].Llamose Estevan Guari ; mi madre nació en San Martín de Valdeiglesias, si bien la casa solar de sus padres fue de la Montañas, de los Velásquez y Canales. Llamose doña Maria de la Canal », Estefanía de la Encarnación, La vida de soror Estephania de la Encarnación, Monja Profesa en el Monasterio de Religiosas Franciscas de Nuestra Me Santa Clara en esta villa de Lerma, 1631, copie ms. XVII'siècle, 268 fol., Madrid, Biblioteca Nacional, ms. 7459, f. 1.

9. Cf. pour l'Italie, Gabriella Zarri, «Monasteri femminili e città (secoli XV - XVIII), in Giorgio Chittolini et Giovanni Miccoli (dirs.), Storia d'Italia. Annali 9. La Chiesa e il potere politico dal Medioevo all'età contemporanea, Turin, Enaudi, 1986, p. 359-429. Pour l'Espagne, par exemple Olegario González Hernández, Una mística abulense, Doña María Vela y Cueto, 1561 - 1617, Ávila, Diputación provincial, 1961. Pour une vue d'ensemble, Isabelle Poutrin, «Las mujeres en el siglo de las reformas religiosas » dans José Fernández Ubiña et Manuel Sotomayor Muro(coord.), Historia del cristianismo, Vol. 3, "El mundo moderno" coord. par Antonio Luis Cortés Peña, Barcelone, Trotta Ediciones, 2006, p. 509-550.

10. Sur le jeu des relations familiales, des liens de clientèle et des affinités entre dévots qui permirent, par exemple, à soeur Mariana de San Agustin (1602-1672) d'être admise dans un des couvents les plus sélects de Madrid, voir I. Poutrin, « L'identité sociale », p. 106.

11. Maria Leticia Sánchez Hernández, El monasterio de la Encarnación de Madrid. Un modelo de vida religiosa en el siglo XVII, Real Monasterio del Escorial, Ediciones Escurialenses, 1986, p. 126-130.

12. Colette Friedlander, «Les pouvoirs de la supérieure dans le cloître et dans le monde du concile de Trente jusqu'à nos jours ", Les religieuses dans le cloitre et dans le monde, Saint-Etienne, CERCOR 1994, p. 239-247.

13. Sur la réforme conventuelle, les courants spirituels et le contexte social à Avila, voir Jodi Bilinkoff, Avila de Santa Teresa, trad. esp., Madrid, Editorial de Espiritualidad, 1993, en part. sur doña María Vela, p. 180-192 ; María Vela y Cueto, Autobiografía y Libro de las Mercedes, éd. par Olegario González Hernández, Barcelone, Juan Flors, 1961 ; Miguel González Vaquero, La Muger fuerte. Por otro titulo la Vida de Doña María Vela, monja de San Bernardo en el convento de Santa Ana de Avila, Madrid, Vuida de Alonso Martin, 1618. Pour d'autres exemples, I. Poutrin, Le voile et la pluma , op. cit., p. 159-167.

14. José Garcia Oro, «Observantes, Recoletos, Descalzos: la monarquía catolica y el reformismo religioso del siglo XVI », Teofanes Egido (coord.), Actas del congreso Internacional Sanjuanista (Avila, 23 - 28 setiembre 1991), t. II : Historia, Junta de Castilla y León, conserjería de Cultura y Turismo, 1993, p. $53-97$.

15. Alban John KRAISHEIMER, «Pouvoirs de la supérieure et réforme monastique au XVII ${ }^{\mathrm{e}}$ siècle : trois exemples cisterciens ", Les religieuses dans le cloître [...], op. cit., p. 257-270. 
16. Anne était la fille de don Juan d'Autriche : Carlos Alonso, Doña Ana de Austria, abadesa del real monasterio de las Helgas (Burgos), Madrid, Biblioteca Nacional, 1990.

17. Francisco Javier Lorenzo Pilar, « Profesiones religiosas femeninas zamoranas en el siglo XVIII ", María Isabelle Viforcos Marines, Jesús Paniagua Pérez (coord.), I Congreso internacional del Monacato Femenino en España, Portugal y América, 1492 - 1992, León, Universidad de León, 1993, vol. 2, p. 373-386. Pour un témoignage de révolte contre les vocations forcées dans l'Italie du XVII siècle, voir Francesca Medioli, L'»inferno monacale » di Arcangela Tarabotti, Turin, Rosenberg \& Sellier, 1990.

18. Ulrike Strasser, «Bones of Contention: Cloistered Nuns, Decorated Relics, and the Contest over Women's Place in the Public Sphere of Counter-Reformation Munich", Archiv für Reformationsgeschichte / Archive for Reformation History, 90, 1999, p. 255-288.

19. Jean Krynen, Saint Jean de la Croix et l'aventure de la mystique espagnole, Université Toulouse - Le Mirail, Presses Universitaires du Mirail, 1990, p. 285-323.

20. Isabelle Poutrin, Le voile [...], op. cit., p. 71-87; Stephen Haliczer, Between Exaltation and Infamy. Female Mystics in the Golden Age Spain, Oxford, Oxford University Press, 2002.

21. Sara Cabibbo et Marilena Modica, La santa dei Tomasi. Storia di Suor Maria Crocifissa (1645-1699), Turin, Enaudi, 1989 ; Giovanna Polin, “Confessione e confessori al femminile : monache e direttori spirituali in ambito Veneto tra '600 e '700", Gabriella Zarri (éd.), Finzione e santità tra medioevo e età moderna, Turín, Rosenberg \& Sellier, 1991, p. 366 - 388 ; Isabelle Poutrin, Le voile [...], op. cit., p. 169-202 ; Jodi Bilinkoff, Related Lives : Confessors and Their Female Penitents, 1450-1750, Ithaca, Cornell University Press, 2005.

22. María Laura Giordano, «Al borde del Abismo: «Falsas santas » e « Ilusas » madrileñas en la vigilia de $1640 »$, Historial Social, n 57, 2007, p. 75-97.

\section{AUTEUR}

\section{ISABELLE POUTRIN}

Université Paris-XII/CREPHE 